\title{
PERCEPÇÃO, CONHECIMENTO E ATUAÇÃO DOS MÉDICOS NO PROGRAMA REDE MÃE PARANAENSE
}

\author{
PERCEPTION AND ACTION OF DOCTORS IN THE \\ PROGRAMA REDE MÃE PARANAENSE
}

\begin{abstract}
Thaís Vanessa Bugs' ${ }^{1}$, Sebastião Caldeira², Gicelle Galvan Machineski ${ }^{3}$, Marlei Fátima Cezarotto Fiewski ${ }^{4}$, Bruna Maria Bugs ${ }^{5}$, Mauren Teresa Grubisich Mendes Tacla ${ }^{6}$, Rosane Meire Munhak da Silva7
\end{abstract}

\section{RESUMO}

Este estudo teve como objetivo compreender como os médicos dos serviços de saúde percebem e atuam no Programa Rede Mãe Paranaense. Para tanto, adotou-se uma pesquisa qualitativa na perspectiva da Fenomenologia Social de Alfred Schütz. Foram realizadas sete entrevistas, no período de junho a agosto de 2014, a partir da análise identificaram-se três categorias: Conhecimento sobre o Programa Rede Mãe Paranaense; Assistência no Programa e Expectativas sobre o programa. A partir dos resultados obtidos verificamos que os médicos possuem conhecimento sobre o programa, mas referem não ter a devida capacitação para atuar no mesmo. Acerca da assistência humanizada, é necessário avaliar as ações conforme sua bagagem de conhecimento profissional. Quanto aos sistemas de informações referem não atuar ou conhecer a importância dos mesmos para o planejamento da assistência, mas gostariam de aprofundar sobre o assunto. Essa pesquisa poderá contribuir para melhorar a capacitação e atuação de profissionais da saúde materno-infantil.

Descritores: Programas Governamentais; Políticas Públicas; Promoção da Saúde.

\begin{abstract}
This study aimed to understand how the health service's doctors realize the "Programa Rede Mãe Paranaense" and how they act in the Program. A qualitative methodology was used from the perspective of Alfred Schutz's social phenomenology. Seven interviews were performed between June and August 2014, by analysing 3 categories were identified: Knowledge about the "Programa Rede Mãe Paranaense", Assistance in the Program and Expectations about the Program. The results show that doctors have knowledge about the program, but the participants said they didn't have a special training to operate it. When referring to humanized care, it's necessary to evaluate the actions considering the professional knowledge that each interviewed has. The information systems are not used and are not considered important during the assistance planning by the professionals, but they would like to know more about it. This study could help in improving the training and performance of professionals in maternal and child health.
\end{abstract}

Descriptors: Government Programs; Public Policies; Health Promotion.
${ }^{1}$ Graduada em Enfermagem pela Universidade Estadual do Oeste do Paraná (UNIOESTE), Cascavel, PR, Brasil.

2 Doutor em Enfermagem pela Universidade de São Paulo (USP), São Paulo, SP, Brasil.

${ }^{3}$ Doutora em Enfermagem pela Universidade Federal do Rio Grande do Sul (UFRGS), Porto Alegre, RS, Brasil.

${ }^{4}$ Mestre em Engenharia de Produção pela Universidade Federal de Santa Catarina (UFSC), Florianópolis, SC, Brasil.

${ }^{5}$ Mestre em Biociências e Saúde pela Universidade Estadual do Oeste do Paraná (UNIOESTE), Cascavel, PR, Brasil.

${ }^{6}$ Doutora em Enfermagem em Saúde Pública pela Universidade de São Paulo (USP), São Paulo, SP, Brasil.

${ }^{7}$ Mestre em Biociências e Saúde pela Universidade Estadual do Oeste do Paraná (UNIOESTE), Foz do Iguaçu, PR, Brasil. 


\section{Introdução}

Em se tratando da saúde da mulher e da criança no âmbito nacional, podemos destacar dentre as políticas e programas do Ministério da Saúde, o Programa de Atenção Integral a Saúde da Mulher, com uma abordagem em todas as fases da vida (adolescência até terceira idade), voltado para planejamento familiar, doenças sexualmente transmissíveis, câncer de mama e de colo de útero, ações no pré-natal, parto e puerpério1.

Uma outra iniciativa foi o Programa de Humanização no Pré-natal e Nascimento que visava reduzir as altas taxas de morbimortalidade materna, perinatal e neonatal ${ }^{2}$, o Programa de Atenção Integral à Saúde da Criança e a Agenda de compromissos para a saúde integral da criança e redução da mortalidade infantil ${ }^{3}$, bem como, os Objetivos do Desenvolvimento do Milênio ${ }^{4}$ como precursores dos mesmos, culminando com o atual Programa Rede Cegonha, cujo objetivo corresponde em garantir à mulher 0 direito à atenção humanizada à gravidez, ao parto (nascimento seguro), puerpério e a criança (desenvolvimento e crescimento saudável) e ao planejamento familiar ${ }^{5}$, fundamentado nos princípios da humanização e assistência do Sistema Único de Saúde (SUS).

Mesmo diante das políticas e programas voltados à humanização à mulher no ciclo gravídico puerperal, as ações humanizadas na assistência e no cuidado somente serão efetivas, se os diversos atores envolvidos, quer seja, na Atenção Primária à Saúde, ou na Atenção Secundária e Terciária, se dispuserem a repensar sua prática cotidiana no que concerne a reorganização dos serviços, bem como, ao uso rotineiro das tecnologias duras, compostas por equipamentos tecnológicos do tipo máquina, normas e estruturas organizacionais, recursos materiais e medicamentos, em detrimento das tecnologias leve-dura como as teorias e a ciência e saberes bem estruturados, como a clínica médica e a epidemiologia, bem como, as tecnologias leves são relacionadas as relações interpessoais como a produção de vínculos, autonomização e acolhimento que abrange a subjetividade e a intersubjetividade entre profissionais e mulher que requer o cuidado de saúde ${ }^{6,7}$.

Dessa forma, levando em consideração as políticas e programas que visam o melhoramento da atenção à saúde, é que surgem as propostas governamentais no âmbito estadual que buscam resolver os problemas na Atenção Primária em Saúde (APS) como o Programa Rede Mãe Paranaense (PRMP) ${ }^{8}$.

Nesse contexto, as Redes de Atenção à Saúde são instituídas sem hierarquia entre os componentes; todos os membros de atenção à saúde são importantes de forma igual; se relacionam horizontalmente; a assistência é continua (nível primário, secundário e terciário); constitui uma atenção integral com intervenções preventivas, curativas, promocionais, paliativas, reabilitadoras e cuidadoras, proporcionam uma atenção adequada, oferecendo serviços seguros, eficientes e efetivos ${ }^{9}$.

Por sua vez a APS no Brasil constitui o fio condutor do cuidado em saúde no SUS, sendo a principal política de governo para a saúde, por meio da Estratégia Saúde da Família (ESF). Oferece atenção para todas as condições, com exceção daquelas consideradas raras, e coordena ou integra a atenção fornecida ao usuário ${ }^{10}$.

No âmbito da APS no Estado do Paraná é importante destacar a implantação no Plano de Governo do Estado para a Saúde 2011 a 2014, do PRMP que visa reduzir os indicadores de mortalidade materna e infantil, com ações de atenção ao pré-natal e à criança, e a vinculação da gestante ao hospital para uma adequada atenção ao parto, conforme o seu protocolo ou linha-guia ${ }^{8}$.

Esse programa foi planejado a partir de dados sobre a Razão de Mortalidade Materna (RMM), a qual considera a qualidade de atenção à saúde da mulher, sendo que, os dados elevados correspondem à prestação de serviço de saúde de forma insatisfatória, desde a assistência pré-natal até o parto e puerpério, bem como do planejamento familiar. De acordo com a Organização Pan-Americana em Saúde e a Organização Mundial de Saúde (OPAS/OMS), a RMM seria baixa quando menor que 20 mortos para cada 100.000 Nascidos Vivos (NV). E entre os anos de2004 a 2008, esse dado no Paraná correspondeu a 62,8/100.000 NV. Aproximadamente $71 \%$ dessas mortes estão relacionadas à atenção prénatal, assistência hospitalar e puerpério, $23 \%$ a causas sociais e $85 \%$ são casos evitáveis ${ }^{8}$.

Nesse sentido, o governo do Paraná está buscando ações para reduzir a morbimortalidade materna e infantil por meio da implantação do PRMP, a qual visa realizar a estratificação de risco da gestante (risco habitual; risco intermediário e alto risco), assim como, para as crianças. E essas ações também compreendem a realização de busca ativa e precoce de crianças (menores de um ano) e gestantes, resultando em um elevado número de pré-natais e na qualidade da assistência, visto que se enfatiza a referência e contra referência entre os diferentes níveis de atenção ${ }^{8}$.Questiona-se então, como os médicos percebem, conhecem e atuam no PRMP.Nesse contexto, destaca-se a relevância em ampliar pesquisas para analisar e avaliar a efetividade da APS. Dessa forma, este estudo teve como objetivo compreender a percepção, atuação e conhecimento dos médicos dos serviços de saúde que atuam no PRMP. 


\section{Metodologia}

Trata-se de um recorte de uma pesquisa multicêntrica intitulada "Análise de implantação e desenvolvimento do PRMP", com financiamento do CNPq, conforme o Edital Universal 14/2013, Processo 474768/2013-9. Tal estudo envolve o Grupo de Pesquisa em Enfermagem Materna e infantil (GPEMI) da Universidade Estadual do Oeste do Paraná, Unioeste, Cascavel, PR, Brasil; a Unioeste de Foz do Iguaçu e a Universidade Estadual de Londrina (UEL). Neste contexto, o estudo partiu da seguinte inquietação: Como os médicos percebem, conhecem e atuam no PRMP?

A presente pesquisa se caracteriza como qualitativa, com suporte na perspectiva da Fenomenologia Social de Alfred Schütz ${ }^{11-13}$, a qual apresenta-se como ciência descritiva, rigorosa, concreta, que mostra, explicita e se preocupa com fenômenos sociais tais como são vividos pelas pessoas. É um referencial que visa compreender a vivência intersubjetiva, ou seja, o mundo com os outros, a relação social, sempre com o olhar voltado para o atendimento das ações dos seres humanos e suas relações sociais dotadas de significado ${ }^{14}$. As investigações qualitativas se preocupam com a realidade, com o universo de significados, bem como com outros motivos, valores e crenças que não podem ser quantificados ${ }^{15}$.

Este estudo foi desenvolvido em cinco municípios, da região oeste do Estado do Paraná, pertencentes à área de abrangência da Décima Regional de Saúde de Cascavel (10a RS), sorteados previamente dentre 25 municípios, seguindo-se os pressupostos da pesquisa fenomenológica que a priori não define a quantidade de sujeitos, primandose pela convergência das informações. Os municípios estudados foram: Cascavel (sede da $10^{a}$ Regional de Saúde), Corbélia; Guaraniaçu; Três Barras do Paraná e Vera Cruz do Oeste.

Os critérios adotados para a inclusão dos sujeitos na pesquisa foram: médicos que atuam no PRMP há pelo menos seis meses. Dessa forma, fizeram parte da pesquisa, sete médicos.

A caracterização da amostra corresponde a: médicos com idades entre 26 e 50 anos. A maioria deles se enquadra como clínico geral (4), dois são ginecologistas e obstetra e um possui especialização de cirurgia geral. 0 tempo médio de formação foi de: oito anos e três meses, sendo que seis relataram que o ensino ocorreu em instituições públicas, sendo um sujeito formado em Cuba, e um no ensino privado. Quanto ao tempo de atuação na Atenção Primária em Saúde variou de: sete meses a 22 anos. Sendo que, quatro médicos trabalham em USF, dois em UBS e um em uma UBS/Centro na saúde, porém presta assistência somente nos casos de urgência e emergência.

As entrevistas foram gravadas no período de junho a agosto de 2014, transcritas na íntegra e encerradas assim que se mostraram convergentes possibilitando a compreensão do cuidado à saúde sob a perspectiva dos médicos.

A organização e a análise dos dados foram realizadas de acordo com os seguintes passos: a) leitura cuidadosa de cada depoimento; b) agrupamento de aspectos significativos presentes nos depoimentos para compor as categorias; $c$ ) análise das categorias, buscando compreender os "motivos porque" e os "motivos para" da ação dos participantes e d) discussão dos resultados com outras evidências científicas pertinentes ao tema em estudo à luz da Fenomenologia Social de Alfred Schüt"

O projeto obteve autorização da direção da $10^{\mathrm{a}} \mathrm{RS}$ e parecer favorável do Comitê de Ética em Pesquisa com Seres Humanos da Universidade Estadual do Oeste de Paraná, sob Protocolo n: 544.107 de 27/02/2014 do CEP/ Unioeste. Todos os sujeitos assinaram o Termo de Consentimento Livre e Esclarecido (TCLE). 0 anonimato dos sujeitos foi preservado, sendo os mesmos denominados como Médico 1 a 7.

\section{Resultados}

As características típicas dos médicos que atuam no PRMP permitiram a análise dos significados em categorias que congregam a compreensão da experiência vivida no tempo passado e presente - "motivos por que" e a intencionalidade da ação - "motivos para"11-13.

Os "motivos por que" são razões enraizadas em experiências passadas que dizem respeito aos atos consumados. Constituem-se na bagagem de conhecimentos que herdamos dos nossos predecessores. Só é possível reconstruir esses motivos em retrospectiva, a partir da reflexão do ato concretizado. Já a orientação para o comportamento futuro constitui-se no "motivo para", que é uma categoria essencialmente subjetiva, pois ainda não aconteceu".

A partir da análise das falas dos sujeitos foram identificadas três categorias que contemplam os "motivos porque" e os "motivos para": Conhecimento sobre o Programa Rede Mãe Paranaense; Assistência no Programa Rede Mãe Paranaense e Perspectivas sobre o Programa Rede Mãe Paranaense. 


\section{Conhecimento sobre o Programa Rede Mãe Paranaense}

O médico por meio de seus depoimentos revela sua bagagem de conhecimentos adquiridos, especificamente os relacionados à implantação, às capacitações oferecidas, aos compromissos, objetivos e indicadores propostos pelo Programa Rede Mãe Paranaense:

[...] é um programa que foi criado para acompanhar as gestantes no pré-natal, fazer um pré-natal correto com exames mais completos, com mais exames do que 0 Ministério preconiza, para detectar mais doenças. (Médico 6)

[...] o programa surgiu do sucesso que foi o Rede Mãe Curitibana, porque reduziu muito a mortalidade materna e infantil [...]ele visa reduzir mortalidade materna e infantil, fazer o acompanhamento da gestante e da criança até os 2 anos de idade e a estratificação de risco, que antes só tinha alto e baixo risco e agora tem intermediário. (Médico 7)

Quando questionado sobre as capacitações, o médico respondeu:

Até hoje ninguém falou pra mim, como funciona o programa. [...] Mas acredito que os enfermeiros foram. (Médico 2)

Não, não fui. [...] eu não recebi nenhuma informação da secretaria nem da regional, a única coisa que recebi foi à cartilha e o que eu sei foi pelo o que eu li. (Médico 7)

\section{Assistência no Programa Rede Mãe Paranaense}

Nesta categoria, o médico fala sobre a realização do pré-natal conforme preconizado pelo Programa Rede Mãe Paranaense, sobre o acesso da gestante ao serviço de saúde e a captação precoce dessa gestante, a estratificação de risco e os indicadores de saúde:

Acredito que sim, eu faço como aprendi na faculdade, conforme preconiza o Ministério da Saúde [...].(Médico 2)

[...] a medicina atende a mulher grávida em geral [...] da mesma forma. [...]. Vou ressaltar que vocês têm muitas doenças na gravidez que no meu país já está erradicada [...]. (Médico 5)

Quanto à captação precoce da gestante, houve as seguintes respostas:

Acontece por meio da vinda delas solicitando o exame de beta HCG, por sorte ela sempre vem no início da gestação, isso é bom porque conseguimos iniciar o pré-natal cedo. (Médico 2)

Na maioria das vezes elas acabam vindo com a suspeita de gravidez, e como os Agentes Comunitários de Saúde (ACS) fazem os acompanhamentos elas sempre acabam encaminhando [...].(Médico 7)

A estratificação de riscos habitual, intermediário e alto risco sempre foi prioridade no cuidado pré-natal:

[...] eu faço, [...] existe o ambulatório de alto risco e essas pacientes daqui da unidade básica são encaminhadas para lá.(Médico 1)

Nas primeiras consultas a gente já pega a carteirinha da Mãe Paranaense, faz as principais perguntas que tem ali [...] e já estratifico como baixo risco, médio ou alto risco. (Médico 6) 
Em relação ao acompanhamento do crescimento e desenvolvimento da criança destacam-se as seguintes falas:

Faço juntamente com os enfermeiros, quando eles realizam a puericultura e notam algo de alterado já fazem o encaminhamento para mim e através de consultas de rotina. (Médico 2)

Geralmente elas vêm por consulta de rotina, a mãe trás, mas a gente tem acesso ao pediatra [...] a criança é bem atendida. (Médico 6)

Quando perguntado ao médico sobre o conhecimento e atuação com os sistemas, o mesmo respondeu:

Para ser bem sincera, eu nem sei o que é isso [...] por que não faço, quem eu vejo fazendo são os enfermeiros. (Médico 2)

Eu sei que [...] tem uma enfermeira responsável na Vigilância Epidemiológica [...] que acompanha tudo isso de perto, [...] se eu vejo alguma coisa notifico e elas têm acesso a essa notificação. (Médico 6)

\section{Perspectivas sobre o Programa Rede Mãe Paranaense}

Em relação aos serviços de saúde, o médico espera:

[...] espero que a gestante seja melhor atendida que seja facilitado o atendimento para ela e para o profissional de saúde também. Isso na verdade é um benefício para todo mundo. (Médico 1)

Que ofereçam suporte e recurso para que possamos realizar o atendimento de forma mais certa possivel. (Médico 7)

As expectativas do médico em relação aos profissionais de saúde:

Espero que [...] sigam o protocolo, porque eu acho que ele é bem elaborado, para ter um objetivo final melhor para todos. (Médico 1)

Uma capacitação seria interessante para a gente conhecer melhor, [...] todos os objetivos, quais são nossas obrigações, o que fazer realmente, o que se espera da gente. (Médico 4)

O que espera dos Sistemas de Informação: (Médico 3)

[...] eu não espero muito, porque eu não mexo muito com os sistemas de informação.

Eu acho que tinha que ser bem mais divulgado para que todos os profissionais tivessem um pouco mais de conhecimento. (Médico 4)

\section{Discussão}

Após a análise das categorias, compreendemos que a maioria dos médicos possui conhecimento sobre a importância do pré-natal e a forma de conduzir as ações voltadas à gestante. Contudo, algumas especificidades do PRMP vigente no Estado do Paraná ainda não são bem claras para esses profissionais.

É importante ressaltar que o PRMP historicamente teve suas origens no programa Rede Mãe Curitibana e Rede Cegonha, norteado por um protocolo que descreve como objetivo e compromisso a realização da captação precoce da gestante e da criança menor de um ano, assim como a busca ativa dessa parcela da população, execução de referência e contrarreferência nos casos de risco intermediário e alto risco, vinculação da gestante ao hospital de referência e 
oferecer um pré-natal de qualidade, bem como acompanhamento e desenvolvimento da criança. Para que os índices de mortalidade materna e infantil diminuam ${ }^{8}$.

Estudo realizado no Espírito Santo que avaliou a assistência do pré-natal conforme preconiza o Programa de Humanização do Pré-Natal e Nascimento (PHPN) e a Rede Cegonha identificou que quando as variáveis, como número de consultas, exame se atividade educativa, são analisadas separadamente elas possuem um índice satisfatório, porém, quando unidos, esses parâmetros apresentam um baixo índice. Sendo assim, as baixas taxas de adaptação do processo pré-natal pode ter como resultado nascimento de crianças prematuras e de baixo peso, além de contribuir para mortalidade materna e perinatal ${ }^{17}$.

Nesse contexto, a formação e a bagagem de conhecimento desses profissionais podem interferir na forma de pensar e de prestar a assistência para as gestantes e crianças. Nesse mesmo pensar, cabe ressaltar a importância da formação e da atuação dos profissionais na área da saúde, e em particular do estudante de Medicina, a partir da perspectiva da integralidade do cuidado ${ }^{18}$.

Um aspecto que chamou atenção nas falas dos médicos foi quanto à capacitação para atuar no PRMP, sendo que alguns afirmaram que mesmo não recebendo atividades de educação permanente disponibilizada pelas Regionais de Saúde, buscaram compreender o programa por conta própria.

No que se refere à relação de cuidado de saúde, os médicos relatam ser necessário reconhecer as carências da gestante e da criança para que se possam identificar quais ações são requeridas por essa população. Para tanto, é fundamental que o médico se utilize da sua bagagem de conhecimentos e vivência profissional no reconhecimento daquelas necessidades, possibilitando ações resolutivas de saúde. É o que Schütz denomina de situação biográfica, que possibilita aos sujeitos compreender o mundo a partir do estoque de conhecimento e experiências, tornando-os competentes para compreender suas ações e sua relação com o mundo ${ }^{12}$.

No entanto, apesar da ausência de capacitação, a assistência ao pré-natal acontece de forma adequada, porém algumas particularidades do PRMP não são abordadas. De acordo com o PRMP a estratificação de risco é dividida em habitual, intermediário e alto, sendo que o atendimento em ambulatório de risco é realizado para ambos gestante e criança. Além disso, a vinculação da gestante e criança é feita no hospital que atenderá suas necessidades, possibilitando um cuidado de qualidade e seguro durante todo o pré-natal, parto, puerpério e às crianças menores de um ano ${ }^{8}$.

Sendo assim, o profissional de saúde necessita apreciar as ações de comunicar-se, ouvir, envolver-se, utilizar de artifício de intersubjetividade para manter uma relação com a gestante, e não apenas tipificá-las conforme o risco gestacional. Essas tipificações ajudam na relação social entre médico e gestante, desde que seja tratada conforme sua necessidade de saúde ${ }^{11-13}$.

Quando questionados sobre a captação da gestante e a importância de que ela ocorra de forma precoce, os médicos destacaram a ida da mulher ao serviço de saúde suspeitando da gravidez e não a busca ativa dessa gestante. Contudo, os serviços de saúde deviam ficar atentos às mulheres em idade fértil, em relação aos sinais e sintomas que algumas vezes não são compreendidos pelas próprias mulheres ${ }^{19}$.

$O$ ideal é que a captação precoce da gestante aconteça antes da $12^{a}$ semana de gestação, podendo ser realizada por qualquer profissional da atenção básica, no entanto, os ACS são de extrema importância nessa tarefa, visto que possuem maior contato com os usuários do serviço de saúde 8 .

No que se refere à classificação de risco, pode-se notar que a mesma ocorre. Contudo, alguns sujeitos utilizam a estratificação de risco em baixo e alto, preconizada pelo Ministério da Saúde. Outros utilizam a classificação recomendada pela Secretária de Saúde do Estado do Paraná(SESA/PR), dividida em: risco habitual, intermediário e alto risco ${ }^{8}$.

A estratificação de risco em três níveis, na qual foi incluído o risco intermediário, foi estabelecida devido ao fato de que a parcela das mulheres que se enquadram nesta categoria, apresenta uma alta taxa de mortalidade materno-infantil. Além disso, as mulheres indígenas e negras apresentam um risco de mortalidade 2,03 vezes maior do que as brancas ${ }^{8}$. 0 que pode ser explicado por características culturais e étnicas, não necessariamente associado a condições de saúde ${ }^{20}$.

Um índice semelhante $(1,97)$ é encontrado nos extremos de idade (menos de 20 e mais de 40 anos). Os valores de risco são similares em mães com pelo menos três filhos vivos em gestação anterior e mães que tiveram pelo menos um filho morto em gestação anterior, caracterizando 2,3 vezes maior e 2,2 vezes maior respectivamente.

Portanto, esses índices apontados pelos documentos da SESA/PR para definir o risco intermediário revela que,se os profissionais de saúde se atentarem para essas características, inúmeras mortes maternas e infantis poderiam ser evitadas ${ }^{21}$. 
A Rede Cegonha ${ }^{5}$ recomenda que aconteça a "Primeira Semana de Saúde Integral" (PSSI). São ações realizadas nos primeiros dias, que visam reduzir a mortalidade materna e infantil, como: vacinação (BCG e hepatite B), triagem neonatal e auditiva, avaliação do aleitamento materno e orientações.

Nesse sentido, o acompanhamento do crescimento e do desenvolvimento da criança necessita ser realizado regularmente, visando identificar alterações o mais rápido possível, possibilitando a assistência precoce ${ }^{22}$.

É importante salientar que uma elevada proporção de mortes infantis ainda é evitável, por meio de pré-natal adequado, parto e cuidados neonatais. Cabe aos profissionais de saúde e aos órgãos governamentais identificar os fatores que causam as mortalidades infantis e proporcionar ações para evitá-las ${ }^{23}$.

Referente às perspectivas dos médicos quanto aos serviços de saúde, importante ressaltar que a humanização do atendimento entre os profissionais com seus usuários de forma qualificada, pode garantir a solução de muitos problemas de saúde. Isso porque, o cuidado, na maioria das vezes, centra-se na assistência técnica, carecendo de investimentos na comunicação, na infraestrutura e no compromisso profissional24.

Sendo assim, a humanização deve acontecer em todos os níveis da assistência, tanto na administração quanto no cuidado, entre profissionais de saúde e usuário, visto que a humanização tem o objetivo de valorizar a intersubjetividade nas relações, pois esta "não se restringe a um conceito abstrato, mas à construção de relações pautadas pela ética de solidariedade, de cuidado com o outro, ela tem que ser efetivada na concretude dos muitos encontros que compõem o sistema"25:135.

A assistência prestada pelos profissionais de saúde deve contemplar a comunicação e o relacionamento interpessoal, atendendo assim as necessidades das gestantes por meio do diálogo entre os sujeitos, numa relação face a face ${ }^{11-13}$, permitindo a troca de informações entre profissional e usuário ${ }^{26}$.

No que se refere aos Sistemas de Informação, os sujeitos desta pesquisa não atuam e não conhecem a importância dos mesmos como aqueles que geram informações para o planejamento da assistência em saúde, e destacam que gostariam de saber mais sobre o tema. Dessa forma, verifica-se a necessidade de formação e capacitação dos médicos para atuarem no contexto dos Sistemas de Informação 27.

Ainda a esse respeito, faz-se necessário pensar na importância da necessidade de capacitação dos profissionais da saúde que assistem diretamente aos usuários do SUS, e que produzem os dados alimentadores dos sistemas, a partir de suas atividades cotidianas do trabalho. As informações geradas a partir de dados de saúde devem estar disponíveis ao público para acesso e uso, e como ferramentas que possam contribuir para melhoria da gestão assistencial prestada pelo SUS ${ }^{28}$.

A utilização adequada dos dados disponíveis nos Sistemas de Informação, possibilitam que os programas implantados pelo Ministério da Saúde, quer sejam desenvolvidos de acordo com as especificidades de cada local, garantindo maior resolutividade nos lugares onde necessitam ${ }^{29}$.

\section{Considerações Finais}

A realização desta pesquisa possibilitou a compreensão de como os médicos pertencentes à área de abrangência da $10^{a}$ Regional de Saúde do Estado do Paraná, percebem e atuam no PRMP a partir da análise de suas motivações.

Sendo assim, o grupo social representado pelos médicos, participantes deste estudo, possui conhecimento sobre os principais objetivos, indicadores e compromissos estabelecidos pelo PRMP e realizam o pré-natal conforme preconizado, identifica as alterações que as gestantes e as crianças, apresenta e realiza os encaminhamentos para os ambulatórios especializados.

Além disso, esse grupo social espera que o cuidado aconteça de forma qualificada. Contudo, se faz necessário maior investimento na capacitação dos profissionais no tocante ao cuidado realizado, bem como sobre a importância dos sistemas de informação, como fonte necessária ao trabalho em equipe, uma vez que os dados devem gerar informações que possibilitem o planejamento das ações de saúde.

A partir das categorias identificadas, o estudo possibilitou a compreensão de que os médicos conhecem e atuam de acordo com as diretrizes do PRMP. No entanto, revelou aspectos que necessitam ser aprimorados como o acesso as capacitações e investimentos no programa. É importante ressaltar que o PRMP encontra-se em fase de implementação, o que a posterior e requer outros estudos que permitam a avaliação do mesmo.

Por fim, o desenvolvimento desta presente pesquisa não esgota as possibilidades de reflexão sobre a temática, mas se coloca como introdução a novas perspectivas de análise que possam contribuir para a compreensão das percepções de profissionais que atuam no PRMP e outros programas semelhantes, a fim de colaborar para a capacitação e consequente melhoria da atenção à saúde da mulher e da criança. 


\section{Referências}

1. Rattner D. Da saúde materno infantil ao PAISM. Tempus, actas de saúde colet [Internet]. 2014 [citado 29 jun 2015 ]. 8 (2): 103-108. Disponível: http://www.tempusactas.unb.br/index.php/tempus/article/view/1460/1314

2. Ministério da Saúde (BR). Programa de Humanização do Pré-Natal e Nascimento (PHPN). Secretaria de Políticas de Saúde. Brasília; 2000. [citado 24 fev 2014]. Disponível: http://bvsms.saude.gov.br/bvs/publicacoes/parto.pdf

3. Ministério da Saúde (BR). Agenda de compromissos para a saúde integral da criança e redução da mortalidade infantil. Secretaria de Atenção à Saúde. Departamento de Ações Programáticas Estratégicas. Brasília; 2004. [citado 20 mar 2014]. Disponível: http://bvsms.saude.gov.br/bvs/publicacoes/agenda_compro_crianca.pdf

4. Organização das Nações Unidas. Objetivos de desenvolvimento do milênio.2000. [citado em 20 fev 2014]. Disponível: http://www.objetivosdomilenio.org.br

5. Ministério da Saúde (BR). Programa Rede Cegonha. Brasília;2011. [citado 01 mar 2014]. Disponível: http://portal. saude.gov.br/portal/saude/gestor/visualizar_texto.cfm?idtxt=37082

6. Santos LM, Pereira SSC, Santos VEP, Santana RCB, Melo MCP. Relacionamento entre profissionais de saúde e parturientes: um estudo com desenhos. R. Enferm. UFSM [Internet]. 2011 [citado 24 jul 2016]; 1(2):225-237. Disponivel:http://periodicos.ufsm.br/reufsm/article/view/2588/1635

7. Jorge MSB, Pinto DM, Quinderé PHD, Pinto AGA, Sousa FSP, Cavalcante CM. Promoção da Saúde Mental: Tecnologias do Cuidado: vínculo, acolhimento, co-responsabilização e autonomia. Ciência \& Saúde Coletiva [Internet]. 2011 [citado 16 jan 2016]; 16(7):3051-3060. Disponível: http://www.scielo.br/pdf/csc/v16n7/05.pdf

8. Secretaria de Estado da Saúde do Paraná (BR). Programa Rede Mãe Paranaense. Linha guia. SESA-PR: Curitiba; 2012. 9. Mendes EV. As redes de atenção à saúde. Organização Pan-Americana da Saúde. Brasília; 2011.

10. Boing AF, VicenziRB,Magajewski F, Boing AC, Moretti-Pires RO, Peres KG et al.Redução das internações por condições sensíveis à atenção primária no Brasil entre 1998-2009.Rev. Saúde Pública [Internet]. 2012 [citado 20 mar 2014];46(2):359-66.Disponível: http://www.scielo.br/pdf/rsp/v46n2/3709.pdf

11. Schütz A, Luckmann T. Lasestructurasdel mundo de la vida. Buenos Aires: Amorrortu; 2003.

12. Schütz A. El problema de larealidad social. Escritos I. $2^{a}$ ed. Buenos Aires: Amorrortu; 2003a.

13. Schütz A. Studios sobre teoría social. Escrito II. $2^{a}$ ed. Buenos Aires: Amorrortu; 2003b.

14. Capalbo C. Fenomenologia e ciências humanas. Aparecida, SP: Idéias e Letras, 2008.

15. Minayo MCS. O desafio do conhecimento. Pesquisa qualitativa em saúde. $9^{a}$ ed. São Paulo: Hucitec; 2004.

16. Caldeira S, Merighi MAB, Muñoz LA, Jesus MCP, Domingos SRF, Oliveira DM. O enfermeiro e o cuidado à mulher idosa: abordagem da fenomenologia social. Rev. Latino- Am. Enfermagem [Internet]. 2012 [citado 28 jan 2013]; 20(5): 1-8.Disponível: http://www.scielo.br/pdf/rlae/v20n5/pt_10.pdf

17. Martinelli KG, Santos Neto ET, Gama SGN, Oliveira AE. Adequação do processo da assistência pré-natal segundo os critérios do Programa de Humanização do Pré-natal e Nascimento e Rede Cegonha. Rev. Bras. Ginecol [Internet].2014 [citado 01 out 2014]; 36(2): 56-64 [acesso em 01 out 2014]. Disponível: http://www.scielo.br/pdf/rbgo/v36n2/0100-7203rbgo-36-02-00056.pdf

18. Costa JRB et al. Formação médica na Estratégia de Saúde da Família: percepções discentes. Rev. bras. educ. med. [Internet]. 2012 [citado 29 jun 2015]; 36 (3): 387-400. Disponível: http://www.scielo.br/pdf/rbem/v36n3/14.pdf

19. Ministério da Saúde (BR). Departamento de Informática do SUS. População Feminina e Idade fértil da Décima Regional de Saúde do Paraná (10ª RS). Brasília; 2012. [Internet] [citado 30 fev 2014]. Disponível: http://tabnet.datasus. gov.br/cgi/tabcgi.exe?ibge/cnv/poppr.def.

20. Batista LE, Escuder MML, Pereira JCR. A cor da morte: causas de óbito segundo características de raça no Estado de São Paulo, 1999 a 2001. Rev. Saúde Pública [Internet]. 2004 [citado 21 jun 2014], vol.38, n.5, pp.630-636. Disponível: http://www.scielo.br/pdf/rsp/v38n5/21749.pdf

21. Figueiró AC, Frias PG, Navarro LM. Avaliação em saúde: conceitos básicos para a prática nas instituições. In: Samico I, Felisberto E, Figueiró AC, Frias PG, organizadores. Avaliação em saúde: bases conceituais e operacionais. Rio de Janeiro: MedBook; 2010.

22. Falbo BCP, Andrade RD, Furtado MCC, Mello DF. Estímulo ao desenvolvimento infantil: produção do conhecimento em enfermagem. RevBrasEnferm [Internet]. 2012[citado 21 set 2014]; 65(1):148-54.Disponível: http://www.scielo.br/pdf/ reben/v65n1/22.pdf 
23. Santos HG, Andrade SM, Silva AMR, Carvalho WO, Mesas AE. Risk factors for infant mortality in a municipality in southern Brazil: a comparison of two cohorts using hierarchical analysis. Cad. Saúde Pública [Internet]. 2012[citado 07 out 2014]; 28(10): 1915-1926. Disponível: http://www.scielosp.org/pdf/csp/v28n10/10.pdf

24. Silva MAG da, Fortunato MM, Costa AWS da, Nascimento EGC do. Humanização na clínica médica: relação usuário - profissional de saúde. Revista Rede de Cuidados em Saúde [Internet]. 2015 [citado 28 jun 2014]; 9(1): 1-15. Disponível: http://publicacoes.unigranrio.com.br/index.php/rcs/article/view/2317

25. Ministério da Saúde (BR). Política Nacional de Humanização.Formação e intervenção. Brasília; 2010. [citado 29jun 2015]. Disponível em: http://bvsms.saude.gov.br/bvs/publicacoes/cadernos_humanizaSUS.pdf

26. Santos MMAS, Saunders C, Baião MR. A relação interpessoal entre profissionais de saúde e adolescente gestante: distanciamentos e aproximações de uma prática integral e humanizada. Ciênc. saúde coletiva [Internet]. 2012 [citado 07 out 2014]; 17(3): 775-786. Disponível: http://www.scielo.br/pdf/csc/v17n3/v17n3a25.pdf

27. Lima AP, Corrêa ACP. A produção de dados para o Sistema de Informação do Pré-Natal em unidades básicas de saúde. Rev EscEnferm. USP [Internet]. 2013 [citado 27 set 2014]; 47(4):876-83. Disponível: http://www.scielo.br/pdf/ reeusp/v47n4/0080-6234-reeusp-47-4-0876.pdf

28. Santos SR, Ferreira JA, Cruz EMMS, Leite EMAM, Pessoa JCS. Sistema de informação em saúde: gestão e assistência no sistema único de saúde. CogitareEnferm [Internet]. 2014 [citado 28 fev 2015]. Disponível: http://ojs.c3sl. ufpr.br/ojs/index.php/cogitare/article/view/35347/23965

29. Andreucci CB, Cecatti JG, Macchetti CE, Sousa MH. Sisprenatal como instrumento de avaliação da qualidade da assistência à gestante.Rev. Saúde Pública [Internet]. 2011[citado 06 out 2014]; 45(5):854-63. Disponível: http://www. scielo.br/pdf/rsp/v45n5/2438.pdf

\section{Thaís Vanessa Bugs}

Endereço para correspondência - Rua: Pinheiro Machado, $n^{\circ} 1197$,

Bairro: Jr. Gisele, CEP: 85905-300, Toledo, PR, Brasil.

E-mail: theesbugs@hotmail.com

Lattes: http://lattes.cnpq.br/4063949808931550

Sebastião Caldeira - calenf3@gmail.com

Gicelle Galvan Machineski - gmachineski@gmail.com

Marlei Fátima Cezarotto Fiewski - marleicf@onda.com.br

Bruna Maria Bugs - brunabugs@yahoo.com.br

Mauren Teresa Grubisich Mendes Tacla - maurentacla@gmail.com

Rosane Meire Munhak da Silva - zanem2010@hotmail.com

\section{Enviado em 22 de setembro de 2015. Aceito em 06 de agosto de 2016.}


